INDO GLOBAL JOURNAL OF

PHARMACEUTICAL SCIENCES

ISSN 2249- 1023

\title{
Phytochemicals: Potential Role in Alzheimer's Disease Therapy
}

\author{
Deeksha Mehtani, Atinderpal Kaur, Shweta Dang* \\ Department of Biotechnology, A-10, Jaypee Institute of Information Technology, Sec-62, Noida, UP, India-201307
}

Address for Correspondance: Shweta Dang, shweta.dang@jiiit.ac.in

\begin{abstract}
Keywords
Alzheimer's

Disease; Anti-

Oxidants; Anti-

Amyloidogenic;

Anti-Alzheimer's

Therapy; Natural

Therapeutics;

Neurodegenerative

Disease;

Phytochemicals.
\end{abstract}

\begin{abstract}
Alzheimer's disease (AD) is an age-related neurodegenerative disease and is one of the most important medical problem affecting the elderly. A number of drugs, including several cholinesterase inhibitors and an N-methyl-D-aspartate receptor antagonist, have been approved for use, but they have been shown to produce diverse side effects and yield relatively low benefits. To overcome the limitations of current therapeutics for $\mathrm{AD}$, extensive research and development is in process to identify drugs that are effective and do not cause any undesirable side effects. Natural products are attractive sources for developing anti-AD agents, because they can provide diverse structural characteristics and biological activities. Certain naturally occurring phytochemicals like resveratrol, huperzine-A, berberine, ginkgolides, bilobalides and many others have received considerable attention as alternative candidates for AD therapy. These phytochemicals have been reported in literature to have the potential to prevent AD because of their anti-amyloidogenic, antioxidative, and anti-inflammatory properties. These phytochemicals also suppress disease progression. The aim to present this review is to summarize the established knowledge about the role of phytochemicals in lowering down the progression of AD. (C) 2016 iGlobal Research and Publishing Foundation. All rights reserved.
\end{abstract}

Conference Proceedings: International Conference on Advances in Plant and Microbial Biotechnology (PMB2017); JIIT, Noida: February 02-04, 2017

Indo Global Journal of Pharmaceutical Sciences( ISSN 22491023 ; CODEN- IGJPAI; NLM ID: 101610675) indexed and abstracted in EMBASE(Elsevier), SCIRUS(Elsevier),CABI, CAB Abstracts, Chemical Abstract Services(CAS), American Chemical Society(ACS), Index Copernicus, EBSCO, DOAJ, Google Scholar and many more. For further details, visit http://iglobaljournal.com 